\title{
Presentation and Analysis of a Diffusion-Velocity Method
}

\author{
G. Lacombe ${ }^{1}$, S. Mas-Gallic ${ }^{1,2}$
}

(1) Université d'Évry-Val d'Essonne, France

(2) C.M.A.P., École Polytechnique, France

lacombe@maths.univ-evry.fr, masgalli@maths.univ-evry.fr

Lagrangian methods were first introduced to solve purely convective problems approximately. These problems include the compressible Euler equations in fluid mechanics, the Vlasov equation in plasma physics. These methods have been extended to non-purely convective problems, to take into account diffusion effects. This has been first done by addition of a random walk to the movement of particles (inclusion of a brownian effect) [1] and the idea was to include most of the effects of the different operators to the dynamics of the particles. Other methods ([2]-[7]) appeared with a different way ot thinking of particles as carriers of information, not only through their position as in the case of random walk, but also through their coefficient. Allowing not only the position but also the weight of the particles to evolve in time, has permitted particle methods to tackle more general operators. Indeed, particles are then no longer considered as clouds of independent points but rather as a set of interacting points.

More recently a new method has emerged: the diffusion-velocity method ([8]-[14]). This method is based on the idea that the diffusion of a scalar quantity $\omega$ has a prefered direction of transport, namely, that of $\nabla \omega$. This remark results in the transformation of a diffusion equation into an advection equation.

In the first section, we present the diffusion-velocity method in the linear case and prove an existence and uniqueness result for a finite time. Additional results are then given for the 1-d case in the second section. In the third section, we consider the case of the 2-d, then 3-d, Navier-Stokes equations.

\section{The linear case}

Consider the linear advection-diffusion equation on the whole $n$-dimensional space with initial condition:

$$
\frac{\partial \omega}{\partial t}+\nabla \cdot(\omega \mathbf{a})-\nu \Delta \omega=0, \quad \omega(., 0)=\omega_{0} .
$$

We assume from now on that the initial condition $\omega_{0}$ is positive. This implies of course that the solution $\omega$ is also positive. In this case, by setting

$$
\mathbf{a}_{d}^{\omega}=\mathbf{a}-\nu \frac{\nabla \omega}{\omega},
$$

equation (1) can be rewritten as a purely transport equation:

$$
\frac{\partial \omega}{\partial t}+\nabla \cdot\left(\omega \mathbf{a}_{d}^{\omega}\right)=0, \quad \omega(., 0)=\omega_{0}
$$


We can now apply a classical particle method. First, we introduce a positive function $\zeta$ such that $\int \zeta(x) d x=1$, and define a "smoothed" velocity $\mathbf{a}_{\zeta}(\omega)$ by

$$
\mathbf{a}_{\zeta}(\omega)=\mathbf{a}-\nu \frac{\omega * \nabla \zeta}{\omega * \zeta}
$$

Here $*$ denotes the convolution product in space. We now replace equation $(2)$ by the following equation:

$$
\frac{\partial \omega}{\partial t}+\nabla \cdot\left(\omega \mathbf{a}_{\zeta}(\omega)\right)=0, \quad \omega(., 0)=\omega_{0},
$$

called the diffusion-velocity transport equation.

The initial condition $\omega_{0}$ is then approximated in the sense of measures by a linear combination of Dirac measures:

$$
\omega_{0}(x) \sim \omega_{0}^{h}(x)=\sum_{p} \alpha_{p} \delta\left(x-x_{p}^{0}\right) .
$$

The numerical method consists in solving the resulting approximated problem:

$$
\frac{\partial \omega_{h}}{\partial t}+\nabla \cdot\left(\omega_{h} \mathbf{a}_{\zeta}\left(\omega_{h}\right)\right)=0, \quad \omega_{h}(., 0)=\omega_{0}^{h}
$$

which reduces to a set of ordinary differential equations. Indeed, one verifies easily that

$$
\omega_{h}(x, t)=\sum_{p} \alpha_{p} \delta\left(x-X_{p}(t)\right)
$$

is an exact solution of equation (4) if the functions $X_{p}$ satisfy:

$$
\frac{d X_{p}}{d t}(t)=\mathbf{a}\left(X_{p}(t), t\right)-\nu \frac{\sum_{q} \alpha_{q} \nabla \zeta\left(X_{p}(t)-X_{q}(t)\right)}{\sum_{q} \alpha_{q} \zeta\left(X_{p}(t)-X_{q}(t)\right)}, \quad X_{p}(0)=x_{p}^{0} .
$$

Two facts must be emphasised: first, the weights $\alpha_{p}$ remain constant in time; second, the numerical solution $\omega_{h}$ is indeed an exact solution of the diffusion-velocity equation. In particular, we can see that, unlike equation (1), the diffusion-velocity equation does not have any smoothing effect.

This method has been known for quite a while now, but to our knowledge no analysis of it has been done yet. As a first result, we can state the following existence and uniqueness property:

Proposition 1 Assume $\omega_{0}$ is regular and that there exist two constants $\alpha, \beta>0$ such that $\alpha<$ $\omega_{0}<\beta$. Assume also that $\mathbf{a}$ is smooth, belongs to $\left(L^{\infty}\left(\mathbb{R}^{\mathrm{n}} \times\right] 0, \mathrm{~T}[)\right)^{\mathrm{n}}$, and finally that $\zeta$ is of class $C^{3}$.

Then there exists $T_{0}<T$ such that equation (3) has a unique solution in $L^{\infty}\left(\mathbb{R}^{\mathrm{n}} \times\right] 0, \mathrm{~T}_{0}[)$.

Proof. The proof is based on a fixed point argument on the functional $\phi$ in $L^{\infty}\left(\mathbb{R}^{\mathrm{n}} \times\right] 0, \mathrm{~T}[)$ that maps any $V \in L^{\infty}\left(\mathbb{R}^{\mathrm{n}} \times\right] 0, \mathrm{~T}[)$ to the unique solution $v$ of the linear advection equation

$$
\frac{\partial v}{\partial t}+\nabla \cdot\left(v \mathbf{a}_{\zeta}(V)\right)=0, \quad v(., 0)=\omega_{0} .
$$


More explicitly,

$$
\phi(V)(x, t)=\omega_{0}(X(0 ; x, t)) \exp \left(-\int_{0}^{t} \nabla \cdot\left(\mathbf{a}_{\zeta}(V)\right)(X(s ; x, t), s) d s\right),
$$

where the characteristic curve $t \mapsto X(t ; x, s)$ is the solution of

$$
\frac{d X}{d t}=\mathbf{a}_{\zeta}(V)(X, t), \quad X(s ; x, s)=x .
$$

We first show that for small enough $T$, the set

$$
\mathcal{A}=\left\{u \in L^{\infty}\left(\mathbb{R}^{\mathrm{n}} \times\right] 0, \mathrm{~T}[) ; \quad \alpha \mathrm{e}^{-1} \leq \mathrm{u} \leq \beta \mathrm{e}\right\}
$$

is stable by the functional $\phi$ and then, again for $T$ small enough, that $\phi$ is a strict contraction on $\mathcal{A}$.

For $i=1,2,3$, set $c_{i}=\|\zeta\|_{W^{i, 1}}$. Now, if $V \in \mathcal{A}$, we have simply

$$
\left|\nabla \cdot\left(\mathbf{a}_{\zeta}(V)\right)\right| \leq 1 / T_{1}
$$

where

$$
\frac{1}{T_{1}}=\|\nabla \cdot \mathbf{a}\|_{0, \infty}+2 \nu e^{4} \frac{\beta^{2}}{\alpha^{2}}\left(c_{2}+c_{1}^{2}\right) .
$$

Thus, if we suppose $T \leq T_{1}$ (and we do so from now on), expression (6) yields immediately

$$
V \in \mathcal{A} \Rightarrow \phi(V) \in \mathcal{A} \text {. }
$$

It remains to study the Lipschitz constant of $\phi$ in $\mathcal{A}$. Let $U, V \in \mathcal{A}$ and set $u=\phi(U), v=\phi(V)$. Then $w=v-u$ satisfies the linear equation

$$
\frac{\partial w}{\partial t}+\nabla \cdot\left(w \mathbf{a}_{\zeta}(V)\right)=f, \quad w(., 0)=0,
$$

with

$$
f=\nabla \cdot\left(u\left(\mathbf{a}_{\zeta}(U)-\mathbf{a}_{\zeta}(V)\right)\right)
$$

This equation can be solved explicitly: we have

$$
w(x, t)=\int_{0}^{t} J(\tau, x, t) f(\tau, x) d \tau
$$

where

$$
J(t, x, s)=\exp \left(\int_{s}^{t} \nabla \cdot \mathbf{a}_{\zeta}(V)(X(\sigma ; x, s), \sigma) d \sigma\right)
$$

and $X$ is given by equation (7). Since $V \in \mathcal{A}$, inequality (8) shows that $|J| \leq e$, and, consequently, that

$$
\|w\|_{0, \infty} \leq T e\|f\|_{0, \infty}
$$

Now, $f$ can be written in terms of convolution products involving $U, V, U-V$ on one side, and derivatives of $\zeta$ on the other side, and in terms of $\nabla u$. Estimating the convolution products poses no problem, and $\nabla u$ is estimated by taking the gradient of expression (6) (with $U$ instead of $V$ ), which leads to

$$
\|\nabla u\|_{0, \infty} \leq\left\|\nabla \omega_{0}\right\|_{0, \infty} e+16 \nu T_{1} e^{4}\left(c_{3} \frac{\beta^{2}}{\alpha}+3 c_{2} c_{1} \frac{\beta^{3}}{\alpha^{2}} e^{2}+2 c_{1}^{2} \frac{\beta^{4}}{\alpha^{3}} e^{4}\right) .
$$


Putting all the pieces together, one obtains the estimate

$$
\|\phi(U)-\phi(V)\|_{0, \infty} \leq T_{1} K\|U-V\|_{0, \infty},
$$

with

$$
\begin{aligned}
K= & 2 \nu c_{1} \frac{\beta}{\alpha^{2}} e^{4}\left\|\nabla \omega_{0}\right\|_{0, \infty} \\
& +16 e^{11}\left(\frac{\beta}{\alpha}\right)^{5}\left(\nu\left(2 c_{2}+2 c_{1}^{2}\right)+2 \nu^{2} T_{1} c_{1}\left(c_{3}+2 c_{2} c_{1}+2 c_{1}^{2}\right)\right) .
\end{aligned}
$$

Thus $\phi$ is a contraction on $\mathcal{A}$ if we suppose $T<\min \left(T_{1}, 1 / K, 1\right)$. This concludes the proof.

\section{The one-dimensional diffusion-velocity equation}

Consider now the simpler case of the one-dimensional heat equation:

$$
\frac{\partial \omega}{\partial t}-\nu \frac{\partial^{2} \omega}{\partial x^{2}}=0, \quad \omega(., 0)=\omega_{0}
$$

Here the "smoothed" diffusion velocity is

$$
a_{\zeta}(\omega)=-\nu \frac{\omega * \zeta^{\prime}}{\omega * \zeta}
$$

and the diffusion-velocity equation (3) becomes

$$
\frac{\partial \omega}{\partial t}-\nu \frac{\partial}{\partial x}\left(\frac{\omega * \zeta^{\prime}}{\omega * \zeta} \omega\right)=0, \quad \omega(., 0)=\omega_{0} .
$$

Assume further that $\zeta$ is chosen even and divisible, i.e. that there exists a function $\chi$ such that $\chi * \chi=\zeta$. For example, functions $\zeta$ of the form

$$
\zeta(x)=\Gamma_{\varepsilon}(x)=\frac{1}{\sqrt{4 \pi \varepsilon}} e^{-x^{2} / 4 \varepsilon}
$$

or

$$
\zeta(x)=C_{\varepsilon}(x)=\frac{1}{\pi} \frac{\varepsilon}{\varepsilon^{2}+x^{2}}
$$

satisfy this properties with, respectively, $\chi=\zeta_{\varepsilon / 2}$ and $\chi=C_{\varepsilon / 2}$. In this situation, the following estimate holds:

Proposition 2 Let $\omega$ be a nonnegative solution of (10) such that $a_{\zeta}(\omega)$ and $\omega * \zeta$ are in $H^{1}(\mathbb{R})$ for every $t \in] 0, T[$. Then, for all $t \in] 0, T[$,

$$
\int|\omega * \chi|^{2} d x \leq \int\left|\omega_{0} * \chi\right|^{2} d x
$$


Proof. Multiplying equation (10) by $\omega * \zeta$ and integrating the result in space yields

$$
\int \frac{\partial \omega}{\partial t}(\omega * \zeta) d x+\nu \int \omega \frac{\left(\omega * \zeta^{\prime}\right)^{2}}{\omega * \zeta} d x=0
$$

and hence

$$
\int \frac{\partial \omega}{\partial t}(\omega * \zeta) d x \leq 0
$$

Now, if $\zeta$ is even, we have

$$
\int \frac{\partial \omega}{\partial t}(\omega * \zeta) d x=\int\left(\frac{\partial \omega}{\partial t} * \zeta\right) \omega d x
$$

and, consequently,

$$
\begin{aligned}
\frac{d}{d t} \int(\omega * \chi)^{2} d x & =\frac{d}{d t} \int \omega(\omega * \zeta) d x \\
& =2 \int \frac{\partial \omega}{\partial t}(\omega * \zeta) d x \leq 0
\end{aligned}
$$

which proves our point.

Some exact solutions of equation (10) Let us mention some cases where an exact solution of equation (10) can be computed.

1. If the initial condition $\omega_{0}$ is a Gaussian, that is if

$$
\omega_{0}(x)=\Gamma_{a_{0}}(x)=\frac{1}{\sqrt{4 \pi a_{0}}} e^{-x^{2} / 4 a_{0}}
$$

and if $\zeta$ itself is a Gaussian as well, say $\zeta=\Gamma_{\varepsilon}$, then one can easily see that the function

$$
\omega(x, t)=\Gamma_{a(t)}(x)
$$

is a solution of equation (10) if the function $t \mapsto a(t)$ satisfies the ordinary differential equation

$$
\frac{d a}{d t}=\nu \frac{a}{a+\varepsilon}, \quad a(0)=a_{0},
$$

or, equivalently, if

$$
\nu t=a(t)-a_{0}+\varepsilon \ln \left(a(t) / a_{0}\right) .
$$

As $\varepsilon$ tends to zero, the smoothed diffusion velocity $a_{\zeta}(\omega)$ tends to $-\nu \frac{\partial \omega}{\partial x} \frac{1}{\omega}$ and the solution of equation (10) can be expected to converge towards the solution of the heat equation (9) with the same initial condition, namely $\omega(x, t)=\Gamma_{a_{0}+\nu t}(x)$. This is actually the case here, at least in $L^{\infty}(\mathbb{R} \times] 0,+\infty[)$, the convergence being of order 1 in $\varepsilon$.

2. If the initial condition $\omega$ is set to be the Dirac measure at the origin: $\omega_{0}(x)=\delta(x)$, and if $\zeta$ is the same Gaussian $\Gamma_{\varepsilon}$, the situation is somewhat different since here, the Dirac measure $\delta(x)$ is a time-independent solution of equation (10) which by no means converges, as $\varepsilon$ tends to zero, towards the solution of the heat equation, which is here $\omega(x, t)=\Gamma_{\nu t}(x)$.

3. If the initial condition is a sum of two Dirac measures:

$$
\omega_{0}(x)=\delta\left(x-x_{0}\right)+\delta\left(x-x_{1}\right)
$$


and if $\zeta$ is the function $C_{\varepsilon}$ defined in (12), equation (5) gives the following solution of the velocitydiffusion equation (10):

$$
\omega(x, t)=\delta\left(x-X_{0}(t)\right)+\delta\left(x-X_{1}(t)\right)
$$

with the two points $x_{0}(t)$ and $x_{1}(t)$ drifting away from each other exponentially fast. More precisely, we have

$$
\left\{\begin{array}{l}
X_{0}(t)=\frac{x_{0}+x_{1}}{2}+\frac{u(t)}{2}, \\
X_{1}(t)=\frac{x_{0}+x_{1}}{2}-\frac{u(t)}{2},
\end{array}\right.
$$

the difference $u(t)=X_{0}(t)-X_{1}(t)$ being a solution of the functional equation:

$$
2 \varepsilon^{2} \ln u(t)+\frac{3}{2} u(t)^{2}+\frac{1}{4 \varepsilon^{2}} u(t)^{4}=4 \nu t+C .
$$

In particular, for large $t, u(t) \sim 2 \sqrt{\varepsilon}(\nu t)^{1 / 4}$.

4. Suppose finally that the initial condition is a difference of two Dirac measures:

$$
\omega_{0}(x)=\delta\left(x-x_{0}\right)-\delta\left(x-x_{1}\right) .
$$

Then, if $\zeta=C_{\varepsilon}$ as above, the solution $\omega(x, t)$ is of the form

$$
\omega(x, t)=\delta\left(x-X_{0}(t)\right)-\delta\left(x-X_{1}(t)\right)
$$

with $X_{0}(t)$ and $X_{1}(t)$ as in (14), with now

$$
\frac{\varepsilon^{2}}{2} u(t)^{2}+\frac{u(t)^{4}}{4}=-4 \nu \varepsilon^{2} t+\frac{\varepsilon^{2}}{2} u(0)^{2}+\frac{u(0)^{4}}{4} .
$$

Here, the two particles come closer to each other at a relative velocity of

$$
\frac{d}{d t}\left(X_{0}-X_{1}\right)=-\frac{2}{X_{0}-X_{1}}\left(\frac{1}{1+\left(X_{0}-X_{1}\right)^{3} \varepsilon^{-2}}\right)
$$

and collide with an infinite velocity at time

$$
t^{*}=\frac{1}{16 \nu \varepsilon^{2}}\left(\left(x_{1}-x_{0}\right)^{4}+2 \varepsilon^{2}\left(x_{1}-x_{0}\right)^{2}\right) .
$$

\section{The two- and three-dimensional Navier-Stokes equations}

Let us first consider the two-dimensional Navier-Stokes equation for an incompressible fluid. Using the Biot-Savart's law, this system can be written in velocity and vorticity variables $\mathbf{u}$ and $\omega$ as follows:

$$
\left\{\begin{array}{l}
\frac{\partial \omega}{\partial t}+\nabla \cdot(\omega \mathbf{u})-\nu \Delta \omega=0 \\
\mathbf{u}=\mathbf{K} * \omega \\
\omega(., 0)=\omega_{0}
\end{array}\right.
$$


Assuming the vorticity never to be zero (this difficulty can nevertheless be overcome since nothing really happens where the vorticity vanishes), the extension of the diffusion velocity method presented above is straightforward, once the diffusion velocity is defined as

$$
\mathbf{u}_{\zeta}(x)=\mathbf{u} * \zeta(x)-\nu \frac{\omega * \nabla \zeta(x)}{\omega * \zeta(x)}
$$

or, equivalently,

$$
\mathbf{u}_{\zeta}(x)=\omega * \mathbf{K}_{\zeta}(x)-\nu \frac{\omega * \nabla \zeta(x)}{\omega * \zeta(x)}, \quad \mathbf{K}_{\zeta}=\mathbf{K} * \zeta
$$

The extension to three dimensions is more problematic. Again written in velocity-vorticity variables, the Navier-Stokes equations now become a system in which both velocity and vorticity are vectors. We could try to apply directly the previous method on each component of the vorticity but this would lead as will be explained in a further remark at the end of this section, to the introduction of a diffusion velocity for each component of the vorticity. This is, of course, not numerically applicable directly since we need to define a velocity for the lagrangian method.

The idea presented here is due to S. Huberson, E. Rivoalen and F. Hauville ([12]-[14]).

The first step is, of course, to write the Navier-Stokes system in velocity and vorticity variables:

$$
\left\{\begin{array}{l}
\frac{\partial \vec{\omega}}{\partial t}+\nabla(\mathbf{u} \otimes \vec{\omega})-(\vec{\omega} \cdot \nabla) \mathbf{u}-\nu \Delta \vec{\omega}=0 \\
\nabla \cdot \mathbf{u}=0, \quad \nabla \times \mathbf{u}=\vec{\omega} \\
\vec{\omega}(., 0)=\vec{\omega}_{0} .
\end{array}\right.
$$

Then, assuming that the vorticity field is not equal to zero, we decompose the vector field $\Delta \vec{\omega}$ into a vector parallel to $\vec{\omega}$ and a vector orthogonal to $\vec{\omega}$ as follows:

$$
-\nu \Delta \vec{\omega}=\mathbf{w}_{t}+\mathbf{w}_{n} .
$$

Now, in order to calculate $\mathbf{w}_{t}$ which is parallel to $\vec{\omega}$, we write it as $\mathbf{w}_{t}=\lambda \vec{\omega}$ and $\lambda$ is found by application of the scalar product by $\vec{\omega}$ of equality (16):

$$
-\nu \Delta \vec{\omega} \cdot \vec{\omega}=\mathbf{w}_{t} \cdot \vec{\omega}+\mathbf{w}_{n} \cdot \vec{\omega} .
$$

This gives $\lambda=-\nu \frac{\Delta \vec{\omega} \cdot \vec{\omega}}{|\vec{\omega}|^{2}}$ and

$$
\mathbf{w}_{t}=-\nu \frac{\Delta \vec{\omega} \cdot \vec{\omega}}{|\vec{\omega}|^{2}} \vec{\omega} .
$$

Proposition 3 The parallel part of $\Delta \vec{\omega}$ defined by (17) can be rewritten as follows

$$
\mathbf{w}_{t}=\nabla\left(\mathbf{u}_{d} \otimes \vec{\omega}\right) .
$$

where

$$
\mathbf{u}_{d}=-\nu \frac{(\nabla \otimes \vec{\omega}) \vec{\omega}}{|\vec{\omega}|^{2}}
$$

is the 3-d diffusion velocity. 
Proof. We choose a basis in which the vector $\vec{\omega}$ is parallel to the vertical axis and an easy calculation thus proves the result as well as the fact that we can rewrite $\mathbf{w}_{t}$ as

$$
\mathbf{w}_{t}=-\nu \nabla\left(\frac{(\nabla \otimes \vec{\omega}) \vec{\omega}}{|\vec{\omega}|^{2}} \otimes \vec{\omega}\right) .
$$

Let us now focus our attention to the vector $\mathbf{w}_{n}$. Since this vector is orthogonal to $\vec{\omega}$, we write it as

$$
\mathbf{w}_{n}=\vec{\omega}_{\nu} \times \vec{\omega} .
$$

and the vector $\vec{\omega}_{\nu}$ has to be determined. The procedure to obtain the value of $\vec{\omega}_{\nu}$ is quite similar to the one previously used. The cross-product by $\vec{\omega}$ of equality (20) leads to

$$
\mathbf{w}_{n} \times \vec{\omega}=\left(\vec{\omega}_{\nu} \times \vec{\omega}\right) \times \vec{\omega}
$$

and, thanks to the classical identity of the double cross-product, to

$$
\mathbf{w}_{n} \times \vec{\omega}=\left(\vec{\omega}_{\nu} \cdot \vec{\omega}\right) \vec{\omega}-|\vec{\omega}|^{2} \vec{\omega}_{\nu}
$$

Finally, imposing its orthogonality with $\vec{\omega}$ defines a unique vector $\vec{\omega}_{\nu}$ and gives

$$
\vec{\omega}_{\nu}=-\frac{\mathbf{w}_{n} \times \vec{\omega}}{|\vec{\omega}|^{2}}=\nu \frac{\Delta \vec{\omega} \times \vec{\omega}}{|\vec{\omega}|^{2}} .
$$

Injecting the previous expressions (16), (18), (20), (19) and (21) in (15), we end up with the following system which replaces the 3-d Navier-Stokes system

$$
\left\{\begin{array}{l}
\frac{\partial \vec{\omega}}{\partial t}+\nabla\left(\left(\mathbf{u}+\mathbf{u}_{d}\right) \otimes \vec{\omega}\right)-(\vec{\omega} . \nabla) \mathbf{u}-\vec{\omega}_{\nu} \times \vec{\omega}=0 . \\
\nabla \cdot \mathbf{u}=0, \quad \nabla \times \mathbf{u}=\vec{\omega}, \\
\mathbf{u}_{d}=-\nu \frac{(\nabla \vec{\omega}) \otimes \vec{\omega}}{|\vec{\omega}|^{2}}, \quad \vec{\omega}_{\nu}=\nu \frac{\Delta \vec{\omega} \times \vec{\omega}}{|\vec{\omega}|^{2}} \\
\vec{\omega}(., 0)=\vec{\omega}_{0} .
\end{array}\right.
$$

Notice that the equation of evolution of $\vec{\omega}$ is now a nonlinear advection equation to which we can apply the previously presented particle method. This form of Navier-Stokes equation does not preserve the vorticity filament structure but does preserve the divergence-free property of the flow.

Actually, in order to compute $\vec{\omega}_{\nu}$, the particle strength exchange method can be applied. This does not, of course, lead anymore to a method with constant weights, but, assuming the nullity of the deformation term, to a method with $|\vec{\omega}|$ constant.

\section{Remarks :}

1.- Let us first mention that the definition of the diffusion velocity $\mathbf{u}_{d}$ is actually a quite natural extension of the scalar case, as will be shown now. We set $\vec{\omega}=\left(\omega_{i}\right)_{i=1,3}$ and consider each component $\omega_{i}$ separately. We can write

$$
-\nu \Delta \omega_{i}=-\nu \nabla \cdot\left(\nabla \omega_{i}\right)
$$

Then, we are led back to the scalar case and assuming the vorticity field to be non-zero, we set

$$
\mathbf{v}_{i}^{d}=-\nu \frac{\nabla \omega_{i}}{\omega_{i}}
$$


and thus get

$$
\Delta \omega_{i}=\nabla \cdot\left(\omega_{i} \mathbf{v}_{i}^{d}\right)
$$

This procedure defines a diffusion velocity in each axis direction and this is not practicable for the particle method. As a matter of fact, we can define a unique diffusion velocity as a convex linear combination of the three different diffusion velocities :

$$
\mathbf{v}_{d}=\sum_{i=1}^{3} \frac{\omega_{i}^{2}}{|\vec{\omega}|^{2}} \mathbf{v}_{i}^{d} .
$$

By a simple calculation (assuming for example that the vector $\vec{\omega}$ is parallel to the vertical axis), we verify that

$$
\sum_{i=1}^{3} \frac{\omega_{i}^{2}}{|\vec{\omega}|^{2}} \mathbf{v}_{i}^{d}=-\nu \frac{(\nabla \otimes \vec{\omega}) \vec{\omega}}{|\vec{\omega}|^{2}}
$$

which means

$$
\mathbf{v}_{d}=\mathbf{u}_{d}
$$

The new diffusion velocity is equal to the diffusion velocity previously defined by (19).

2.- Let us mention the work of Strickland-Kempka-Wolfe ([11]). They assume that some of the terms in the decomposition of the vorticity are negligible. This assumption which is actually not clearly satisfied from a theoretical point of view enables them to restrict themselves to the two-dimensional case for the computations and they obtain good results.

\section{References}

1 Chorin A. J.: Numerical study of slightly viscous flows, J. Fluid Mech., 57, 785-796 (1973).

2 Rehbach, C.: Calcul numérique d'écoulements tourbillonnaires tridimensionnels instationnaires avec nappes tourbillonnaires, La Recherche Aérospatiale, 5, 289-298 (1977).

3 Cоттет G.H., Mas-Gallic S.: A particle method to solve the Navier-Stokes system, Numer. Math., 57, 805-827 (1990).

4 Choquin, J.P., Huberson S.: Particle simulation of viscous flows, J. Computers and Fluids, 17, n2, 397-410 (1989).

5 Mas-Gallic S., Raviart P.A.: Particle approximation of convection-diffusion equations, Internal Report, 86013, Analyse Numérique, Université Pierre et Marie Curie (1986).

6 Degond P., Mas-Gallic S.: The weighted particle method for convection-diffusion equation, Math. Comp., 53, 485-525 (1989).

7 Fishelov D.: A new vortex scheme for viscous flows, J. Comp. Phys., 86, 211-224 (1990).

8 Fronteau J., Combis X.: Hadronic J., 7, 911-940 (1984).

9 Degond P., Mustieles F.J.: Approximation of diffusion equations by deterministic convections of particles, SIAM J. on Scientific and Statistical Computing, 11, 293-310 (1990).

10 Ogami Y., Akamatsu T.: Viscous flow simulation using the discrete vortex model - the diffusion velocity method, Computers and Fluids, 19, 433-441 (1991).

11 Strickland J.H., Kempka S.N., Wolfe W.P.: Viscous diffusion of vorticity using the diffusion velocity concept, in ESAIM Proceedings, Vortex flow and related numerical methods II, 1, 135-151 (1996).

12 Rivoalen E.: PhD Thesis, Le Havre (1994).

13 Huberson S., Rivoalen E., Hauville F.: Simulation numérique des équations de Navier-Stokes 3D par une méthode particulaire, C.R. Acad. Sci. Paris, 324, Série IIb, 543-549 (1997).

14 Huberson S., Rivoalen E.: Numerical simulation of axisymmetric viscous flows by means of particle method (submitted). 